\title{
LA-UR-18-31769
}

Approved for public release; distribution is unlimited.

Title: $\quad$ Toward an understanding of aging in plutonium from direct measurements of stored energy

Author(s): $\quad$ Migliori, Albert

Ennaceur, Susan M.

Intended for: internal seminar

Issued: 
Disclaimer:

Los Alamos National Laboratory, an affirmative action/equal opportunity employer, is operated by Triad National Security, LLC for the National Nuclear Security Administration of U.S. Department of Energy under contract 89233218CNA000001. By approving this article, the publisher recognizes that the U.S. Government retains nonexclusive, royalty-free license to publish or reproduce the published form of this contribution, or to allow others to do so, for U.S. Government purposes. Los Alamos National Laboratory requests that the publisher identify this article as work performed under the auspices of the U.S. Department of Energy. Los Alamos National Laboratory strongly supports academic freedom and a researcher's right to publish; as an institution, however, the Laboratory does not endorse the viewpoint of a publication or guarantee its technical correctness. 


\section{Toward an understanding of aging in plutonium from direct measurements of stored energy}

- S.M. Ennaceura, A. Migliorib*

- aAlamo Creek Engineering, Santa Fe, NM 87506

- 'Material Physics and Application Division, Los Alamos National Laboratory, Los Alamos, NM 87545

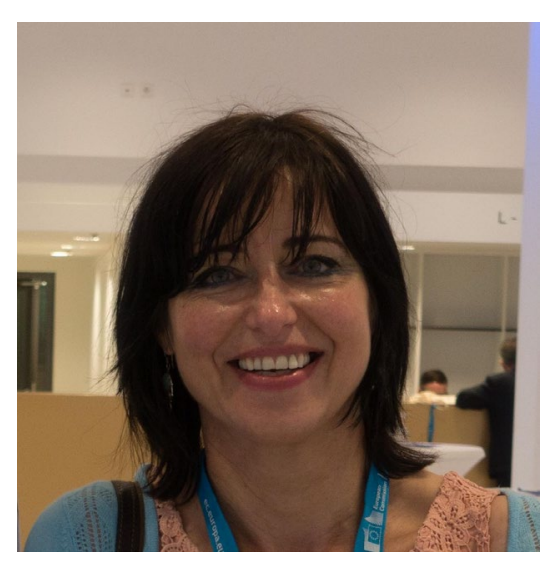

Sue Ennaceur

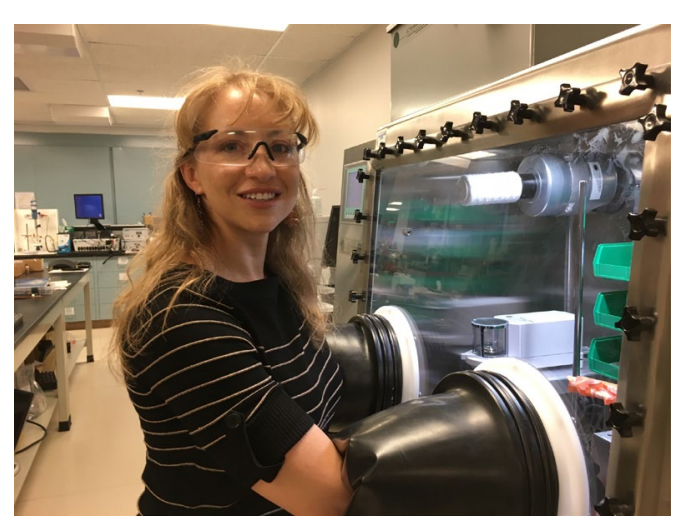

Alex Marchi

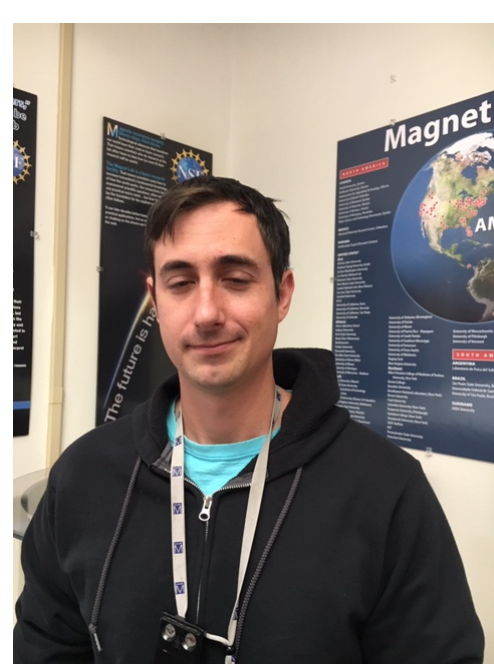

Mark Wartenbe

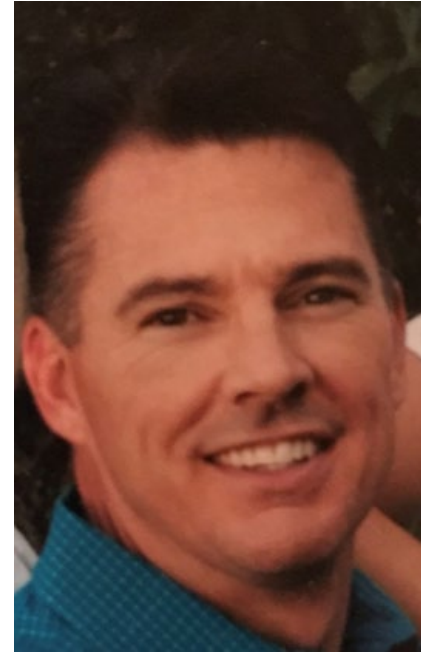

Troy Semelsberger
Albert Migliori

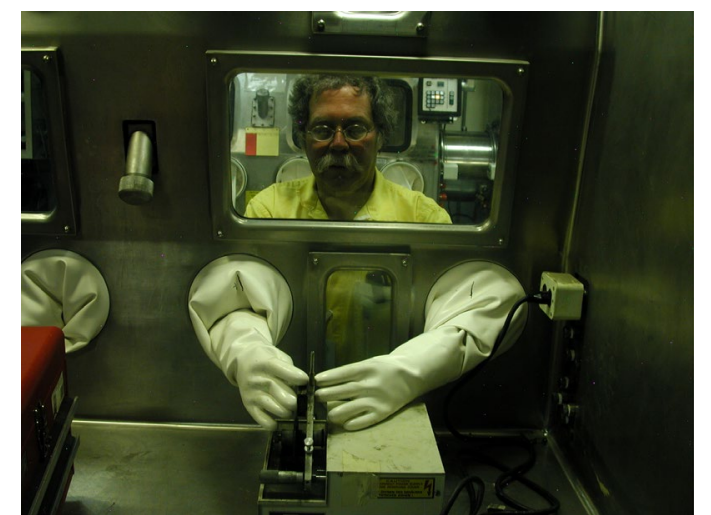




\section{What we will show}

- Plutonium has a propensity to make short bonds. Pu is its own impurityLawson calls it a self-intermetallic.

- What we are sure of and what is suspect in observing aging.

- Short-bond Pu defects lead to a workable quantitative model of aging.

- The model is falsifiable; we make testable predictions. 

overlooked in attempts to understand damage
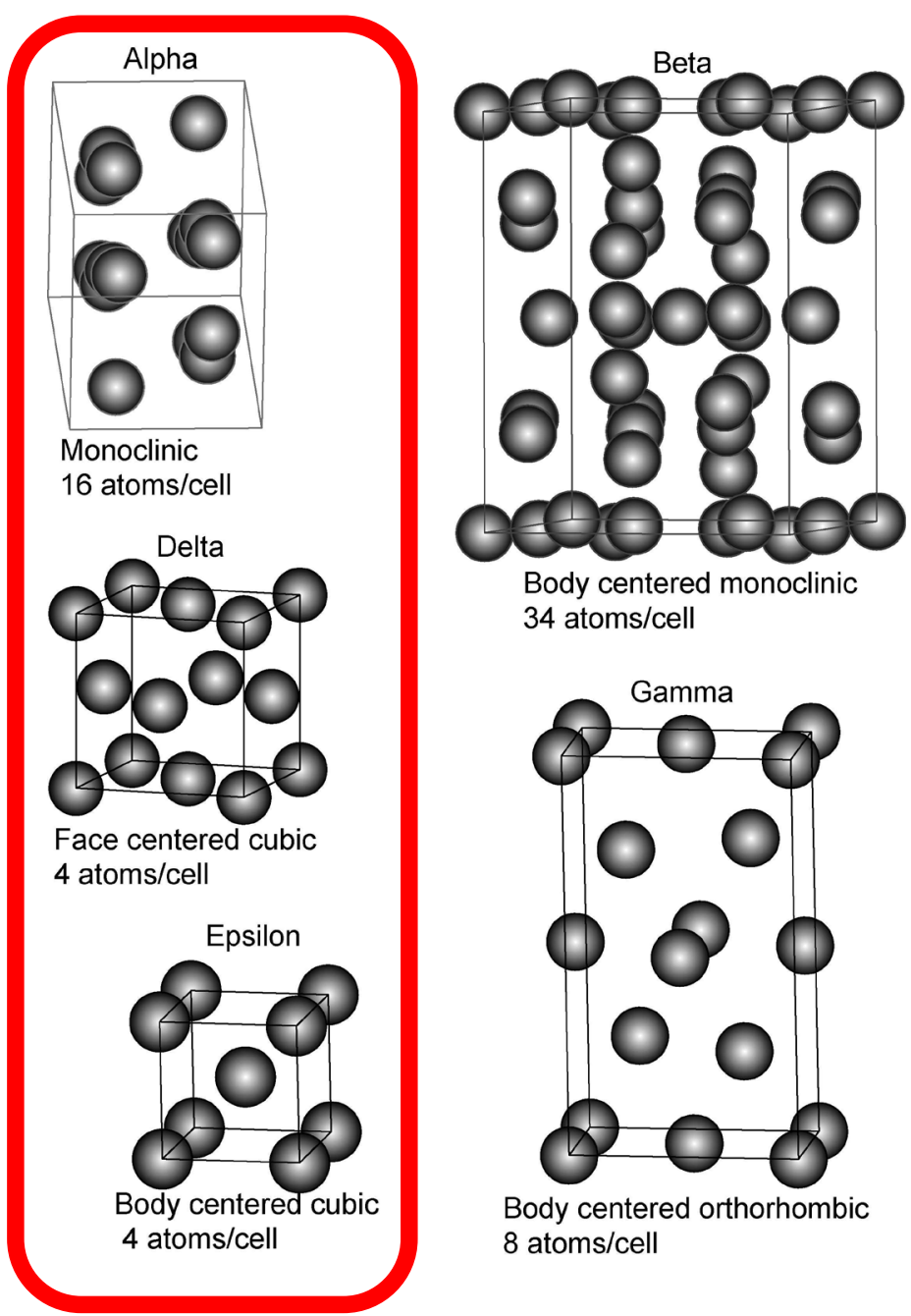


\section{Pu as its own impurity (1)}

The thermodynamically-stable $\alpha$-phase at room temperature has two groups of bonds

Table 10. 'Short' and 'long' bonds (Å)

\begin{tabular}{lccccccc}
$\begin{array}{c}\text { Atom } \\
\text { type }\end{array}$ & \multicolumn{2}{c}{ Short bonds } & \multicolumn{2}{c}{ Long bonds } & \multicolumn{2}{c}{ All bonds } \\
No. & Range & No. & Range & No. & Mean \\
lenth & \\
I & 5 & $2 \cdot 57-2 \cdot 76$ & 7 & $3 \cdot 21-3 \cdot 71$ & 12 & $3 \cdot 10$ \\
II & 4 & $2 \cdot 60-2 \cdot 64$ & 10 & $3 \cdot 19-3 \cdot 62$ & 14 & $3 \cdot 21$ \\
III & 4 & $2 \cdot 58-2 \cdot 66$ & 10 & $3 \cdot 24-3 \cdot 65$ & 14 & $3 \cdot 18$ \\
IV & 4 & $2 \cdot 58-2 \cdot 74$ & 10 & $3 \cdot 26-3 \cdot 42$ & 14 & $3 \cdot 13$ \\
V & 4 & $2 \cdot 58-2 \cdot 72$ & 10 & $3 \cdot 24-3 \cdot 51$ & 14 & $3 \cdot 19$ \\
VI & 4 & $2 \cdot 64-2 \cdot 74$ & 10 & $3 \cdot 21-3 \cdot 65$ & 14 & $3 \cdot 22$ \\
VII & 4 & $2 \cdot 57-2 \cdot 78$ & 10 & $3 \cdot 30-3 \cdot 51$ & 14 & $3 \cdot 15$ \\
VIII & 3 & $2 \cdot 76-2 \cdot 78$ & 13 & $3 \cdot 19-3 \cdot 71$ & 16 & $3 \cdot 32$
\end{tabular}

- At room temperature, Pu wants to be $\alpha$.

- Unlike other fcc and bcc metals, defects and radiation damage in $\delta$-plutonium have an "escape route" into short bonds.

- Short bonds can absorb radiation damage and thermal defects easily. 


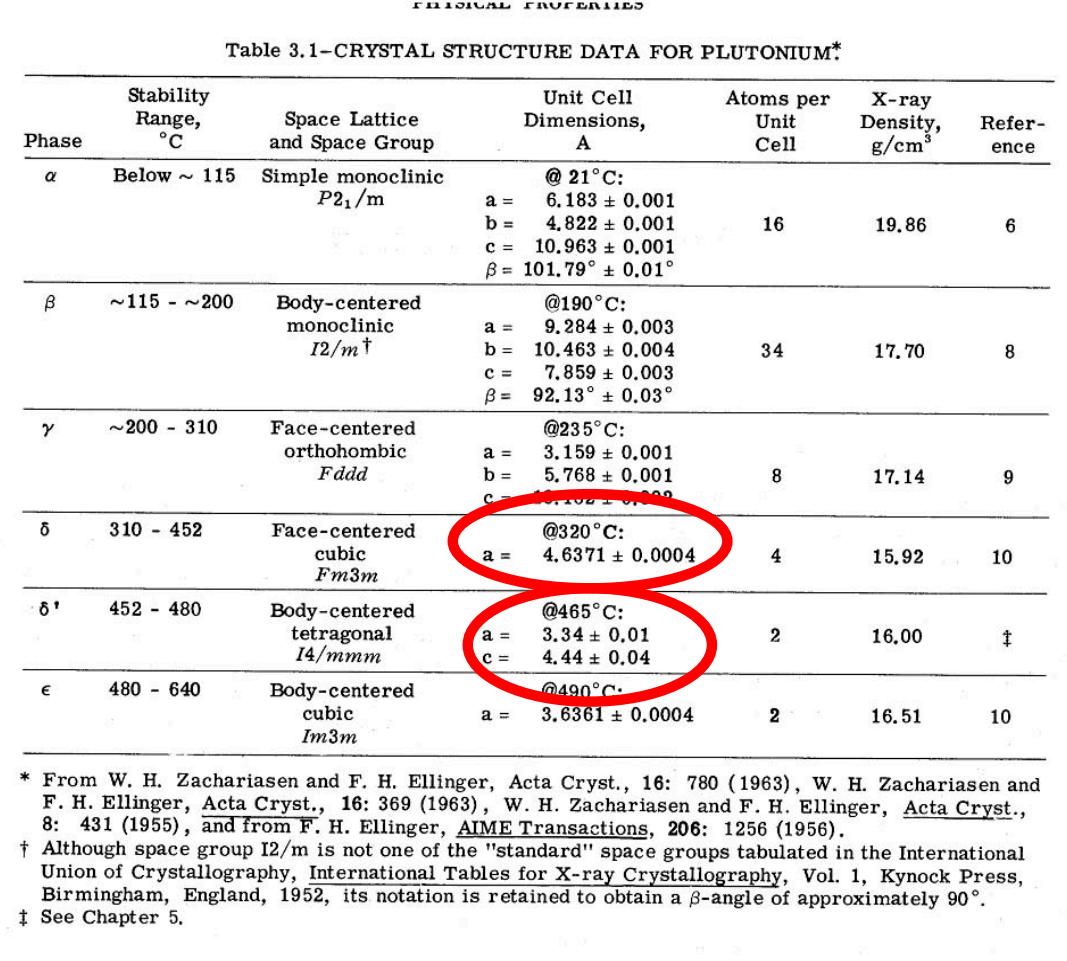

- $\delta^{\prime}-\mathrm{Pu}$ is never observed by itself. Either the $\delta$ or $\varepsilon$ phase is always present.

- The errors in determining structure are huge.

- The latent heat is essentially zero.

- The tetragonal distortion is tiny.

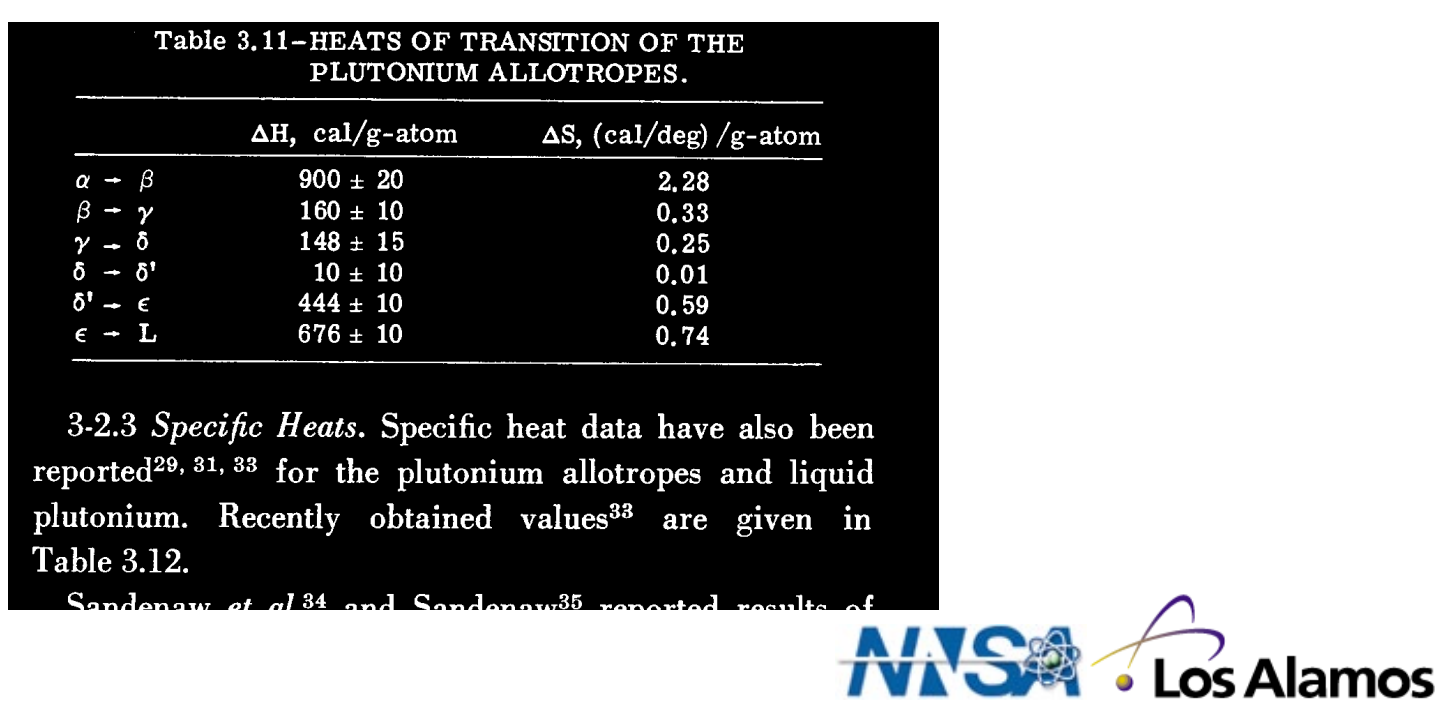


Pu as its own impurity (3)

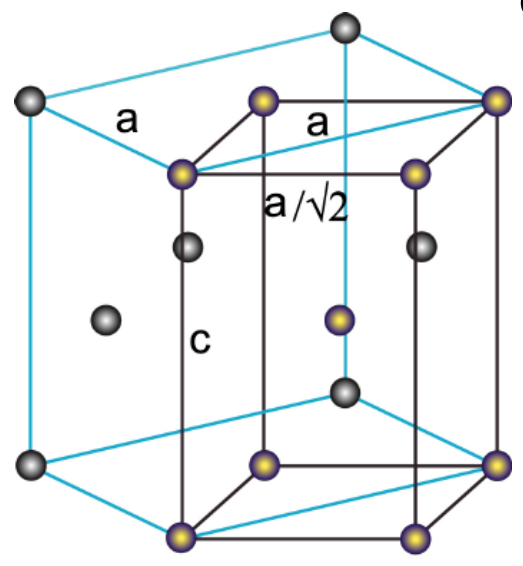

$\delta^{\prime}$-is a strain-induced distortion leading to short-bonds.

$f c c$ and bct

representation

$\delta \quad$ fcc $0.4637 \mathrm{~nm}$

bet $0.4637 \mathrm{~nm} 0.328 \mathrm{~nm}$ ratio $\sqrt{2}$

$\delta^{\prime} \quad$ bet $0.444 \mathrm{~nm} 0.334 \mathrm{~nm}$ ratio $1.06 x \sqrt{2}$

$\varepsilon \quad$ bcc $0.36361 \mathrm{~nm}$

$\delta$-Pu is very soft (softer than $\mathrm{Pb}$ ) and has an especially soft 110 shear mode.

The ratio $c_{44} / c^{*}=7$ is the shear anisotropy. It is the largest for any fcc metal.

Poisson's ratio is about 0.43 along the soft direction, making Pu nearly like a liquid when squeezed in this direction.

$\delta$ ' may just be a manifestation of strain field. But this is one easy route to short bonds in the fcc structure. 


\section{What is suspect (1)}

\section{the Frenkel-pair picture of radiation damage in $\delta$-plutonium}

From LA Science, a string of assumptions based on assuming $\mathrm{Pu}$ is an ordinary fcc metal:

"assessment of radiation damage in gallium stabilized $\delta$-phase plutonium by assuming it is a ... "normal" face-centered-cubic (fcc) metal(s)... it has lattice defect properties consistent with those of other fcc metals such as nickel, copper, and austenitic stainless steels."

"To calculate the number of displacements, we need to know the so-called displacement energy, $E_{\mathrm{d}}, \ldots$

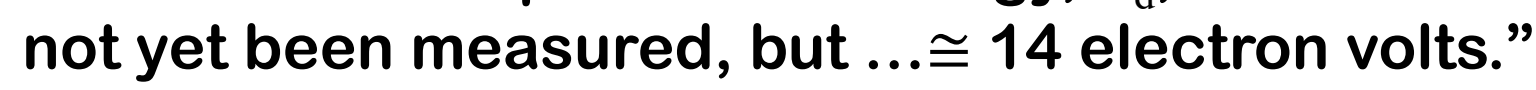

This is huge!! It takes about $100 \mathrm{meV} /$ atom to melt.

This yields, btw, $1 \mathrm{dpa}=14.7$ years based on cascading and questionable assumptions. dpa is not a useful number.

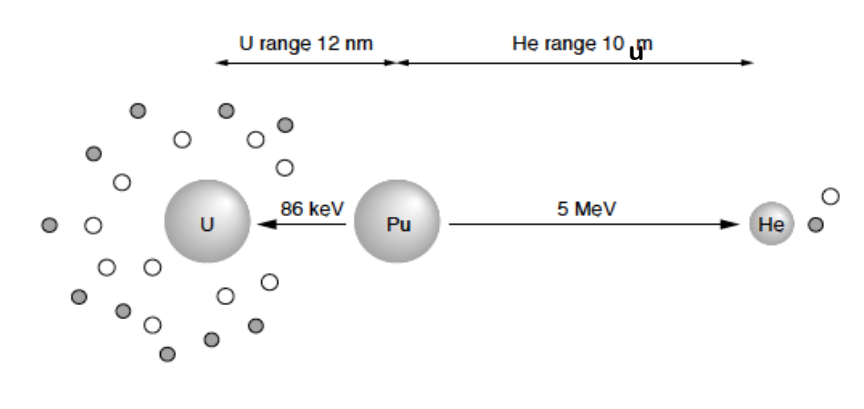


Before we get to the next bit...

\section{CURVE-FITTING METHODS} AND THE MESSAGES THEY SEND
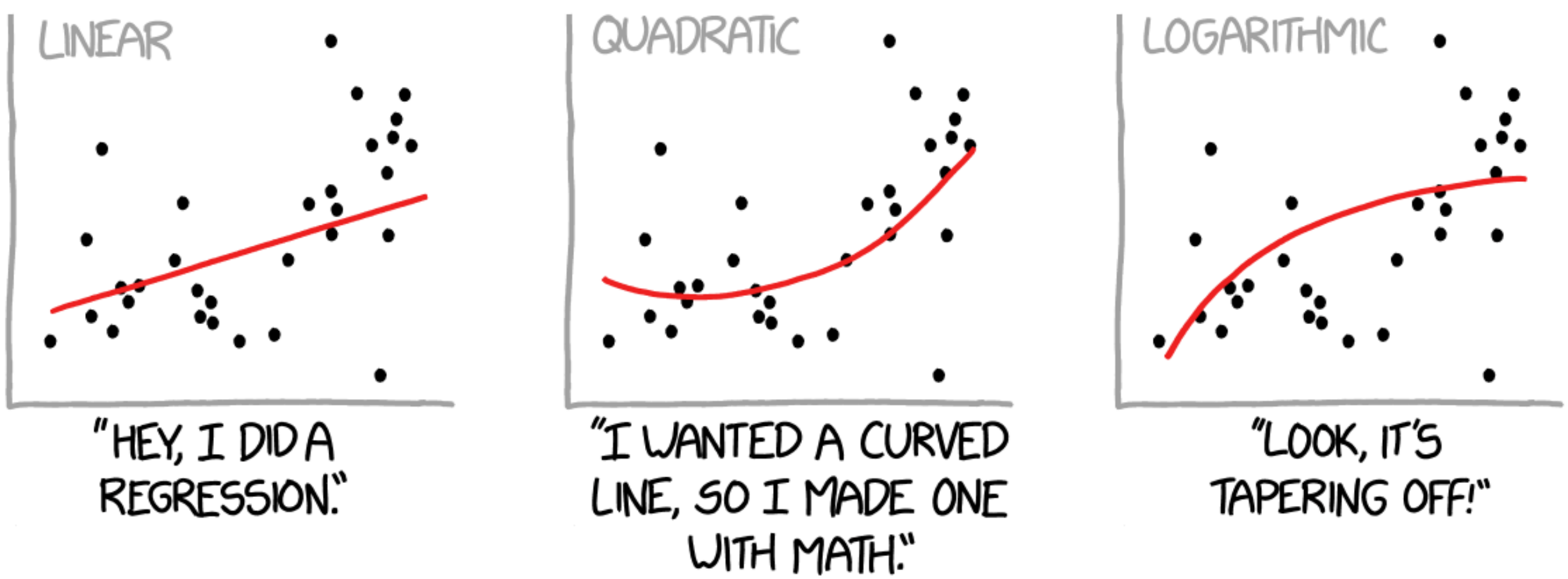
diffraction studies do not show density decreases with age

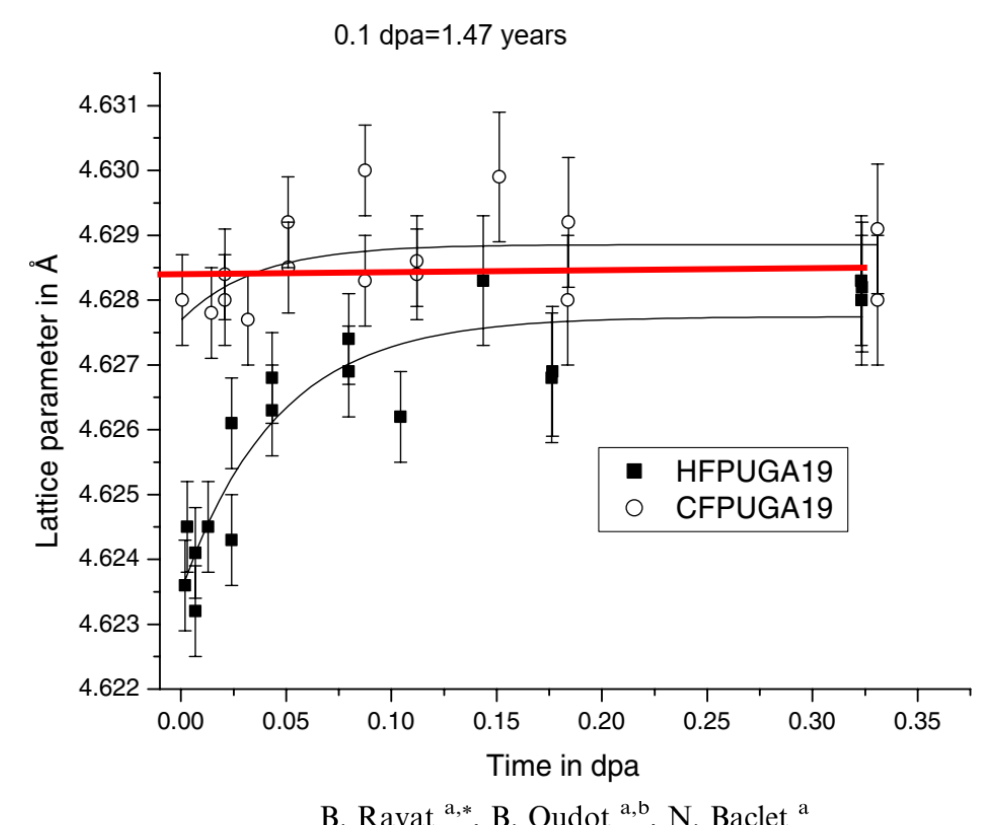

Of the several sample of $\delta$-plutonium used for this study, some had excessive Ga, some were not "homogenized".

The only result for a well-homogenized 2 at. \% Ga sample (open circles) shows no change (my fit in red) in lattice parameter with time.

The lattice parameter is not useful for

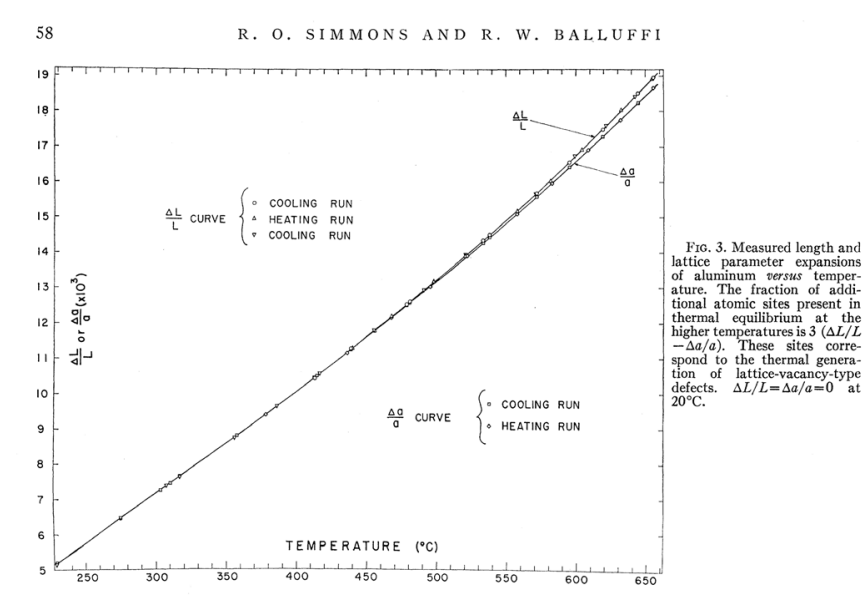
determining density in the presence of defects.

The difference between actual density and $x$-ray density is well studied and thermallpy activated. 
What is suspect (3)

density ands dilatometry studies do not show with any certainty that density decreases with age

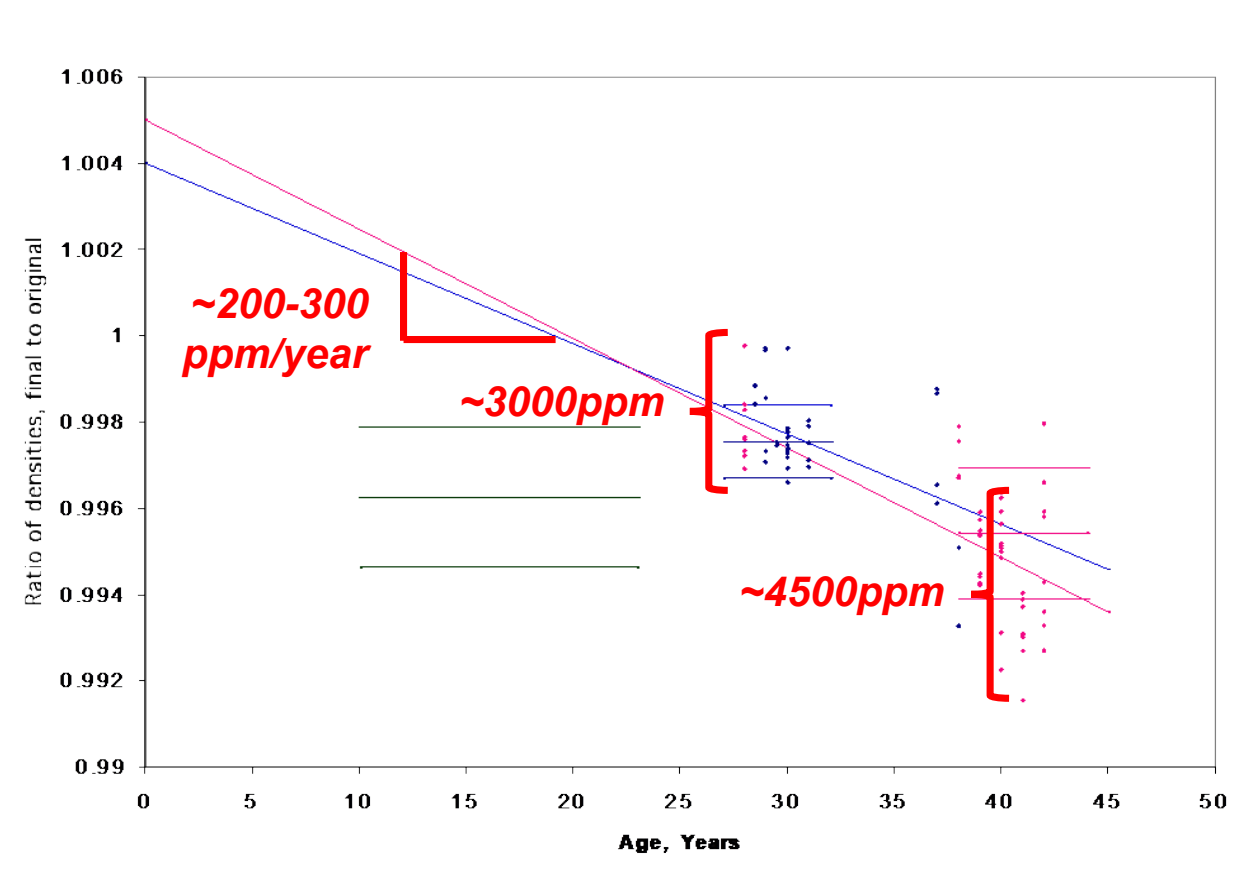

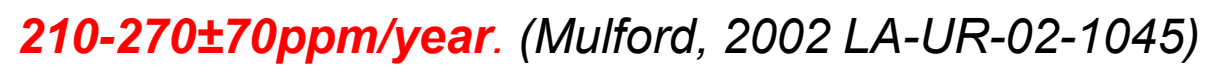
Robbie did the best she could but unknown systematic variations remain.

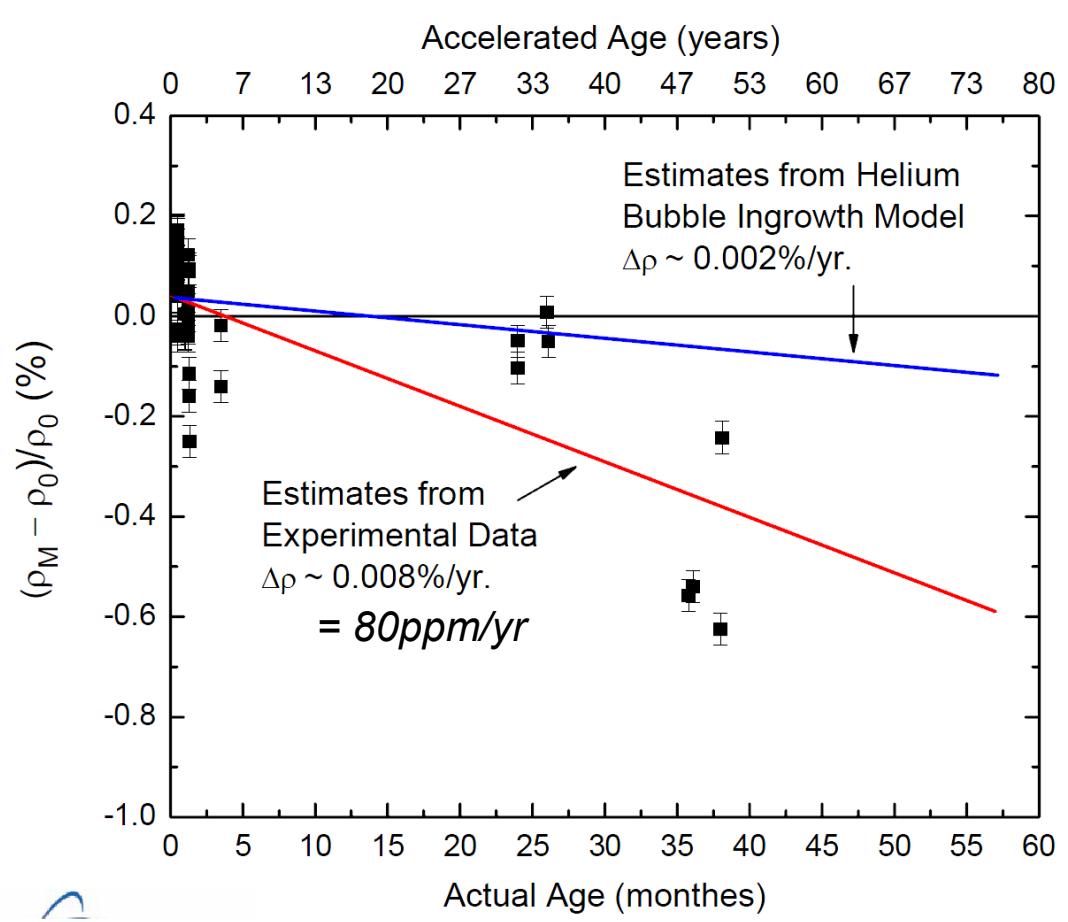

(Freibert, 2005 LA-UR-05-9007) too much scatter to be of use. 
A missing systematic correction to immersion density measurements

$1.5 \mathrm{~mW} / \mathrm{g}$

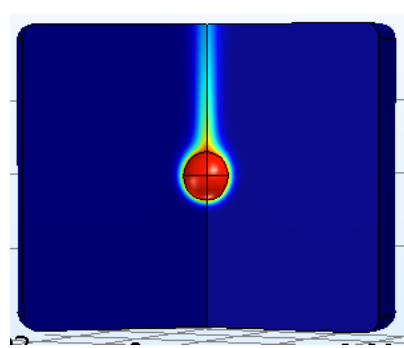

$2 \mathrm{~mW} / \mathrm{g}$

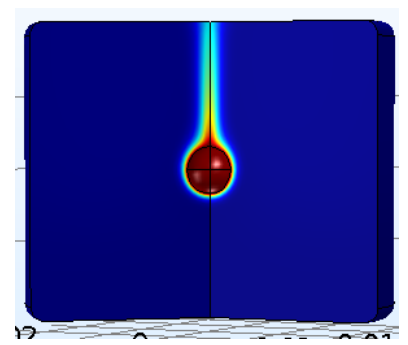

$2.5 \mathrm{~mW} / \mathrm{g}$

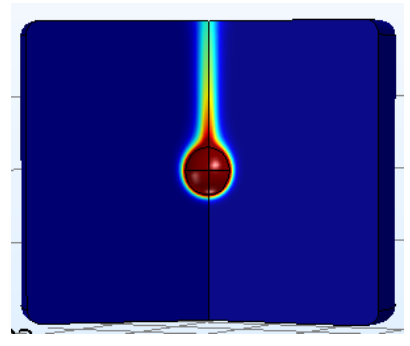

Heat Output of WG-Pu-239
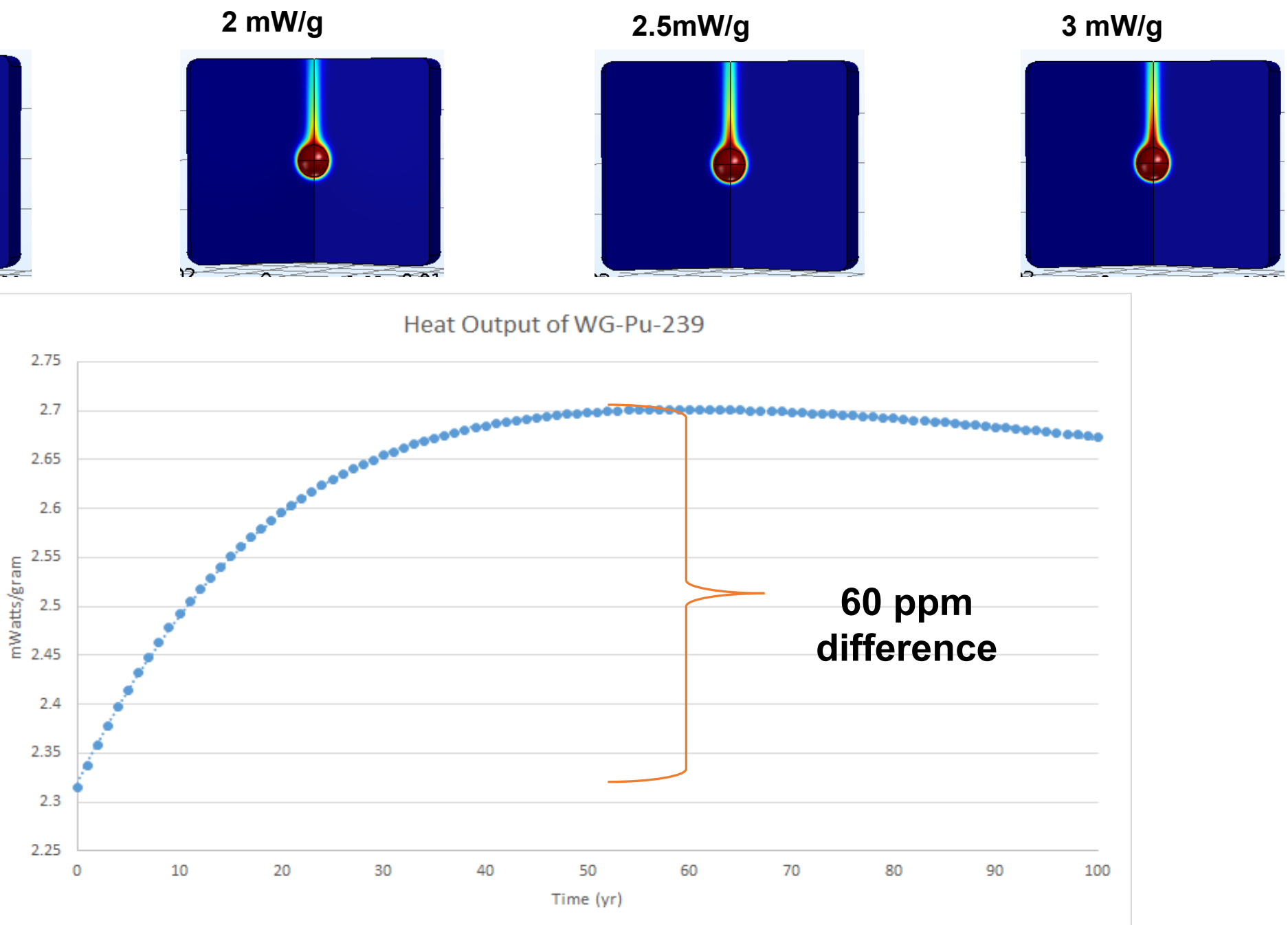


\section{What we know (1)}

Two thermodynamic properties are very unusual
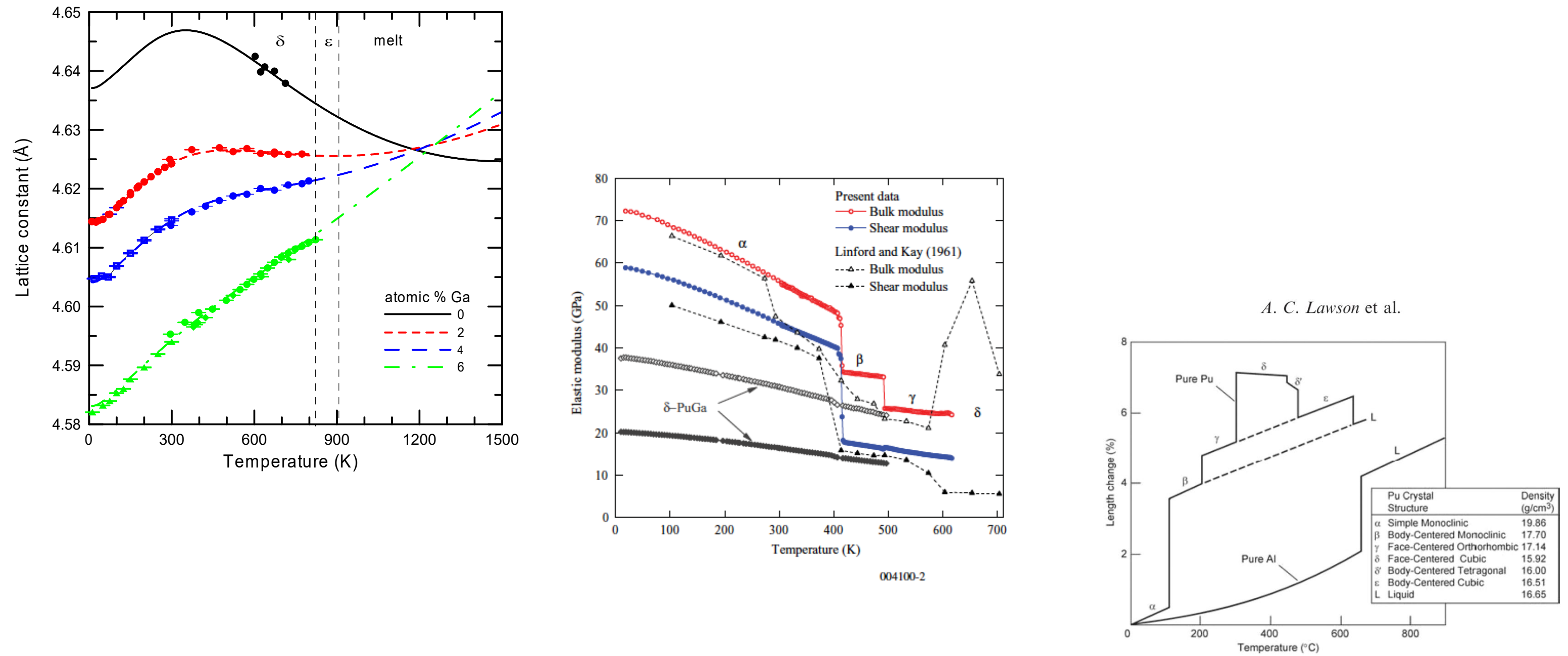
The elastic moduli of both $\delta$ - and $\alpha$-Pu increase with age at about the same rate
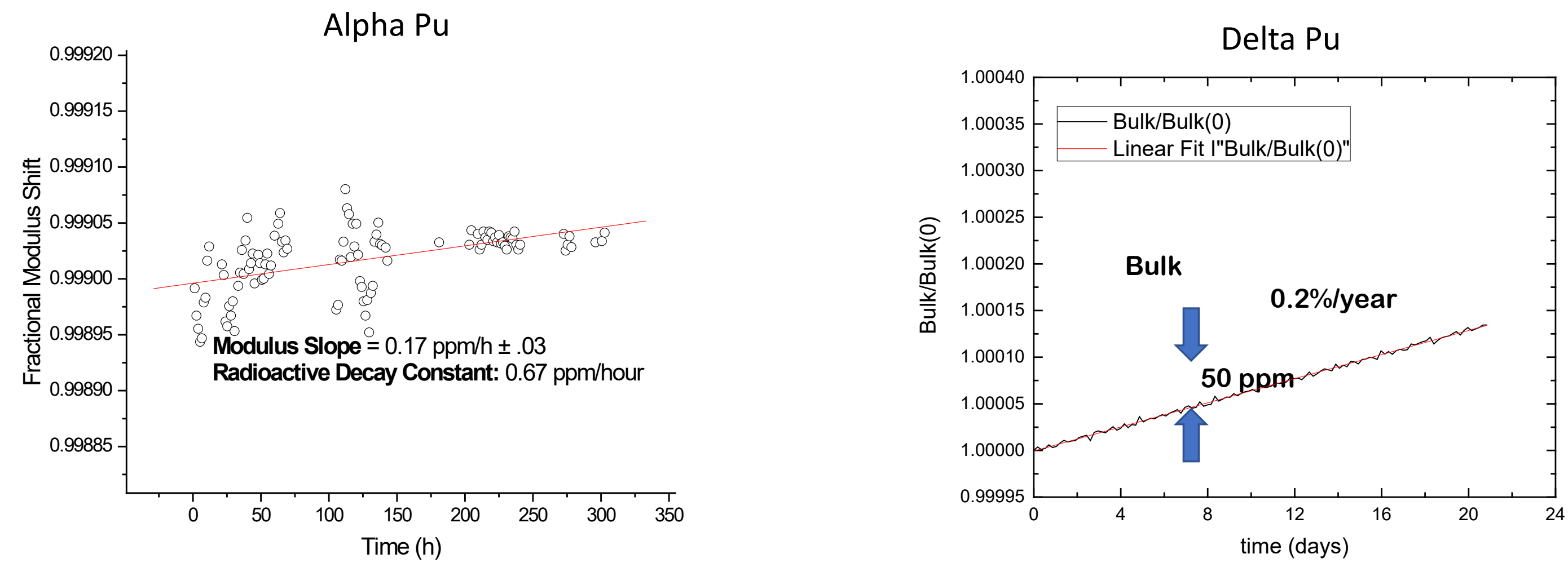


\section{What we know (3)}

- Exponential decay factor for ${ }^{239} \mathrm{Pu}$ is $1.1 \times 10^{12} \mathrm{~s}$, or about 34,770 years.

- In one year, about $30 \mathrm{ppm}$ of $\mathrm{Pu}$ is converted to $\mathrm{U}$ and $\mathrm{He}$ atoms.

- $85 \mathrm{keV}$ of energy to the uranium, 5.2 Mev to the alpha, and a spectrum of mostly low energy gamma rays with the most probable at $51 \mathrm{keV}$.

- Most decay energy is converted immediately to heat, about $1.9 \mathrm{~mW} / \mathrm{g}$. Some energy remains trapped. It is that thermallyrecoverable energy that we are trying to understand.

- Most of the alpha particle energy is deposited to electrons and then to heat: little local heating, $10 \mu \mathrm{m}-$ long track.

- Most of the $U$ energy is deposited directly as heat. 


\section{How differential scanning calorimetry works}

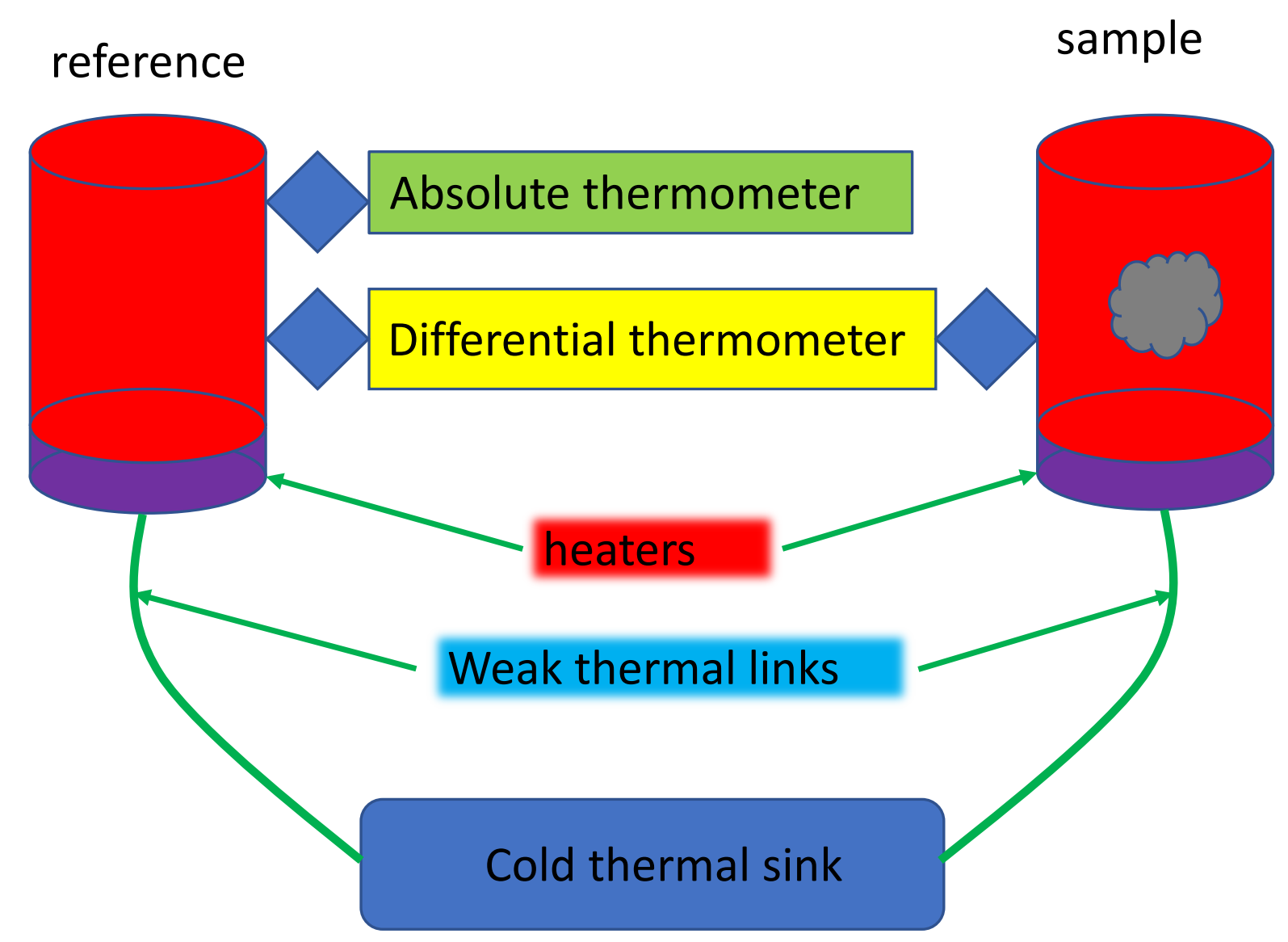

Measures heat required to keep sample at same temperature as reference while reference temperature is increased at constant rate 


\section{What we know (4)}

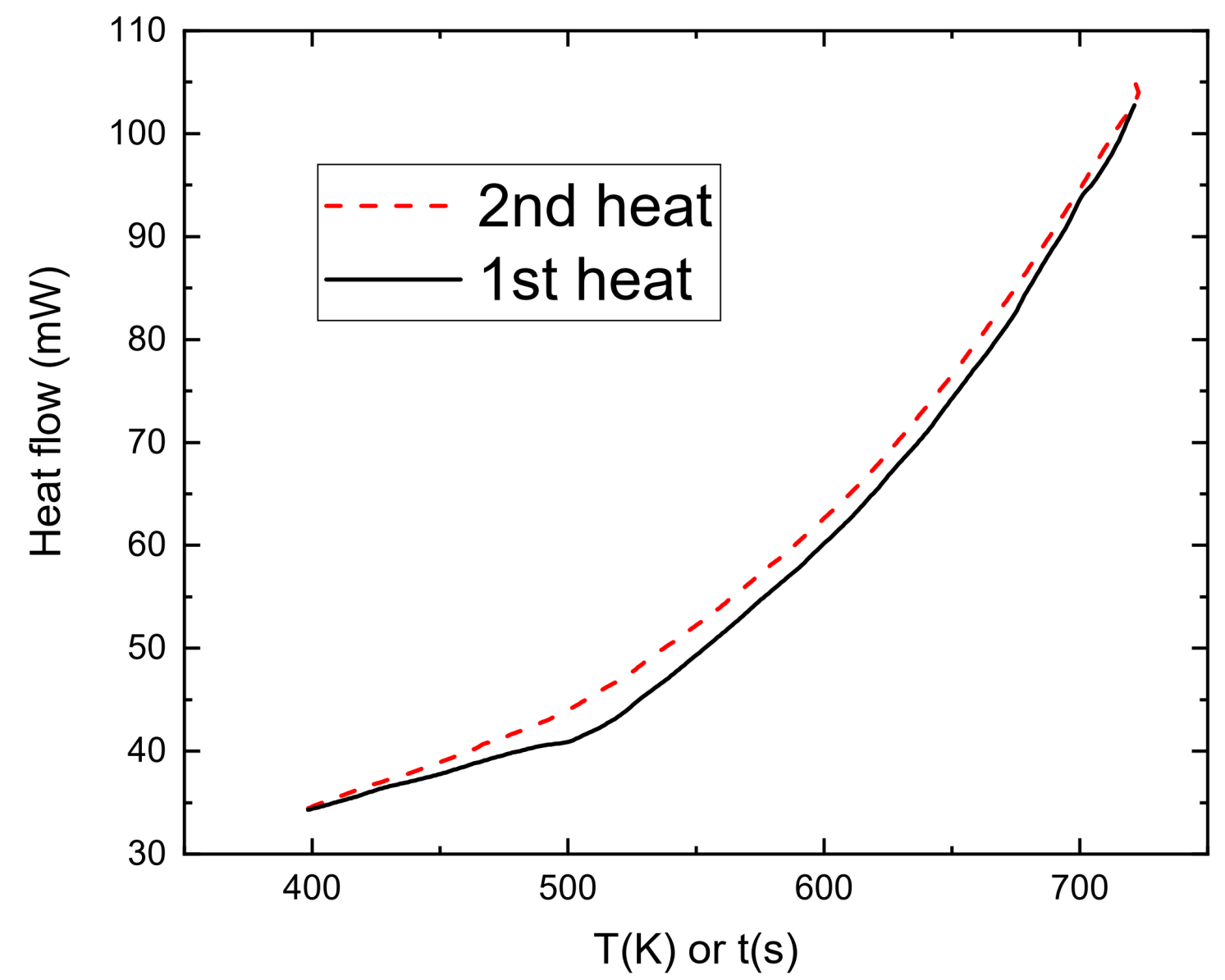




\section{What we know (5)}
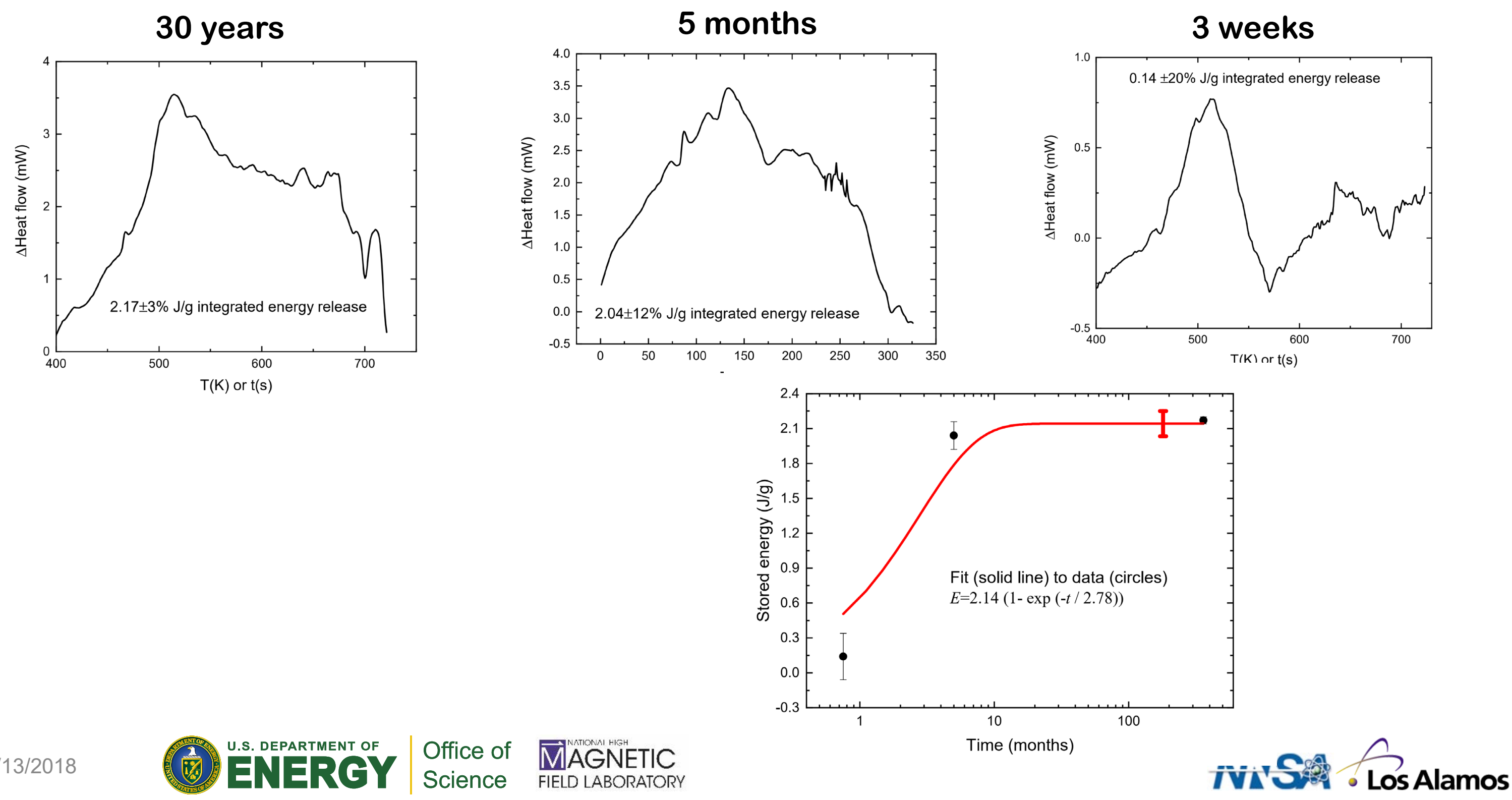
Neglecting duplicates we have:

- 12 long bond-short bond pairs using 24 atoms.

- 17 long bonds.

- Thus about $60 \%$ of the unit cell is long bond-shortbond pairs.

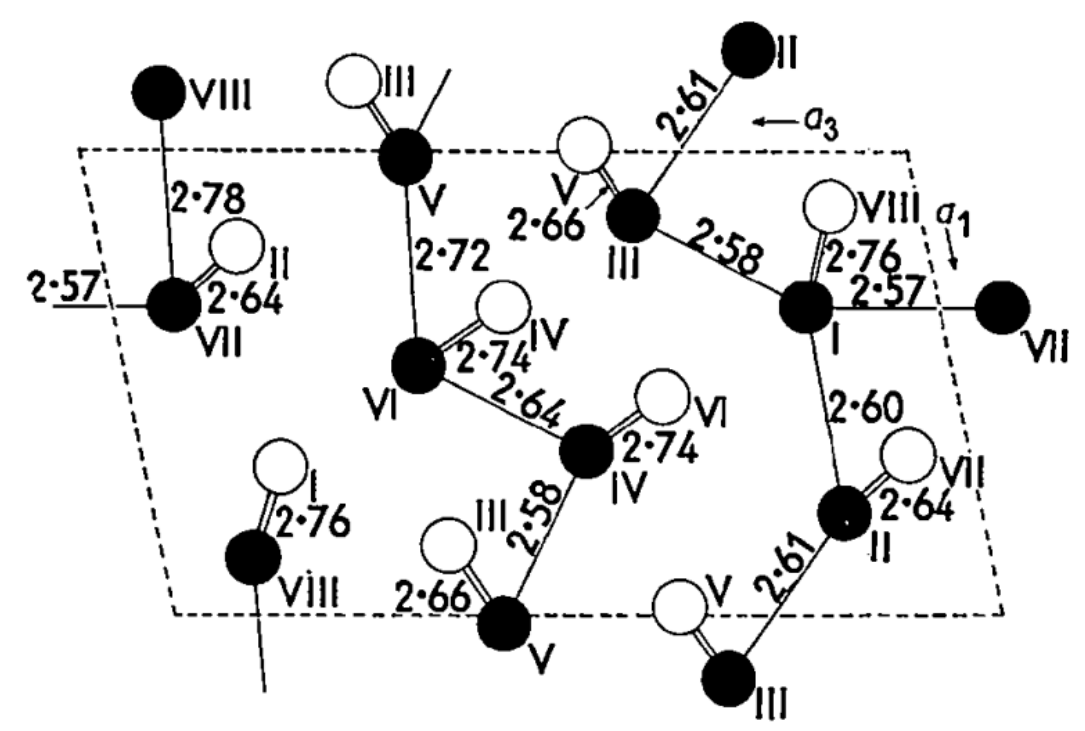

Fig. 3. Projection of the structure on the $(010)$ plane. Filled circles represent atoms at $y=\frac{1}{4}$, open circles atoms at $y=-\frac{1}{4}$. Only the short bonds are indicated in the projection. 


\section{$\alpha-\mathrm{Pu}$ as $\delta$ - Pu with long-bond short-bond $\mathrm{Pu}$ impurities (2)}

How can radiation damage induce bond defects and what do they do?

- From decay flash heating, regions of about $100 \mathrm{~nm}$ radius must produce pressure pulses from thermal expansion, just what is needed to compress the alloy toward the $\alpha$-phase, destabilizing locally $\delta-\mathrm{Pu}$.

- Such an effect can also change locally an $\delta$-Pu specimen by creating excess short bonds.

- Both the $U$ and the flash heating can produce short bonds, so that two time scales are expected.

- $50 \mathrm{~J} / \mathrm{g}$ (100 meV/atom) takes Pu from ambient temperature to melt.

- The energy is deposited locally, so each decay can raise $10^{6} \mathrm{Pu}$ atoms above melt.

- In about 20 days, the $U$ recoil deposits $50 \mathrm{~J} / \mathrm{g}$, raising all the atoms, more or less, above melt. This is the DSC time scale. 
Considering the as an impurity, we can find what the bulk modulus $B$ of the impurity must be to make adding that impurity to $\delta$ - $\mathrm{Pu}$ into $\delta$ - Pu by making the pressure everywhere the same for a small volume change $d V$.

$$
\begin{gathered}
B_{a} \frac{d V}{N_{l}+N_{s}}=B_{d} \frac{d V_{d}}{N_{l}}=B_{s} \frac{d V_{s}}{N_{s}} \quad \begin{array}{c}
N_{s} \text { short-bond } \\
\text { long-bond pairs }
\end{array} \quad N_{l} \text { long-bonds } \\
B_{s}=110 \mathrm{GPa}
\end{gathered}
$$


We do the same for the density of the short-bond long-bond pairs:

$$
\begin{aligned}
& \rho_{\mathrm{a}}=\left(\rho_{\mathrm{d}} * N_{l}+\rho_{s} * N_{s}\right) /\left(N_{l}+N_{s}\right) \\
& \rho_{\mathrm{s}}=22 \mathrm{~g} / \mathrm{cc} .
\end{aligned}
$$


- We know from DSC study that at 8 years, all the thermallyrecoverable effects of damage have saturated.

- The only quantity linear at 8 years is the rate of decay product production ( $\mathrm{U}$ or $\mathrm{He}$ ) of $30 \mathrm{ppm} / \mathrm{yr}$.

- We know the bulk modulus of the short-bond long-bond pairs and so we know how many must be produced per $U$ to get the RUS results $(0.2 \% / y r$ or $2000 \mathrm{ppm} / \mathrm{year})$.

- Thus about 50 short-bond long-bond pairs per decay are retained.

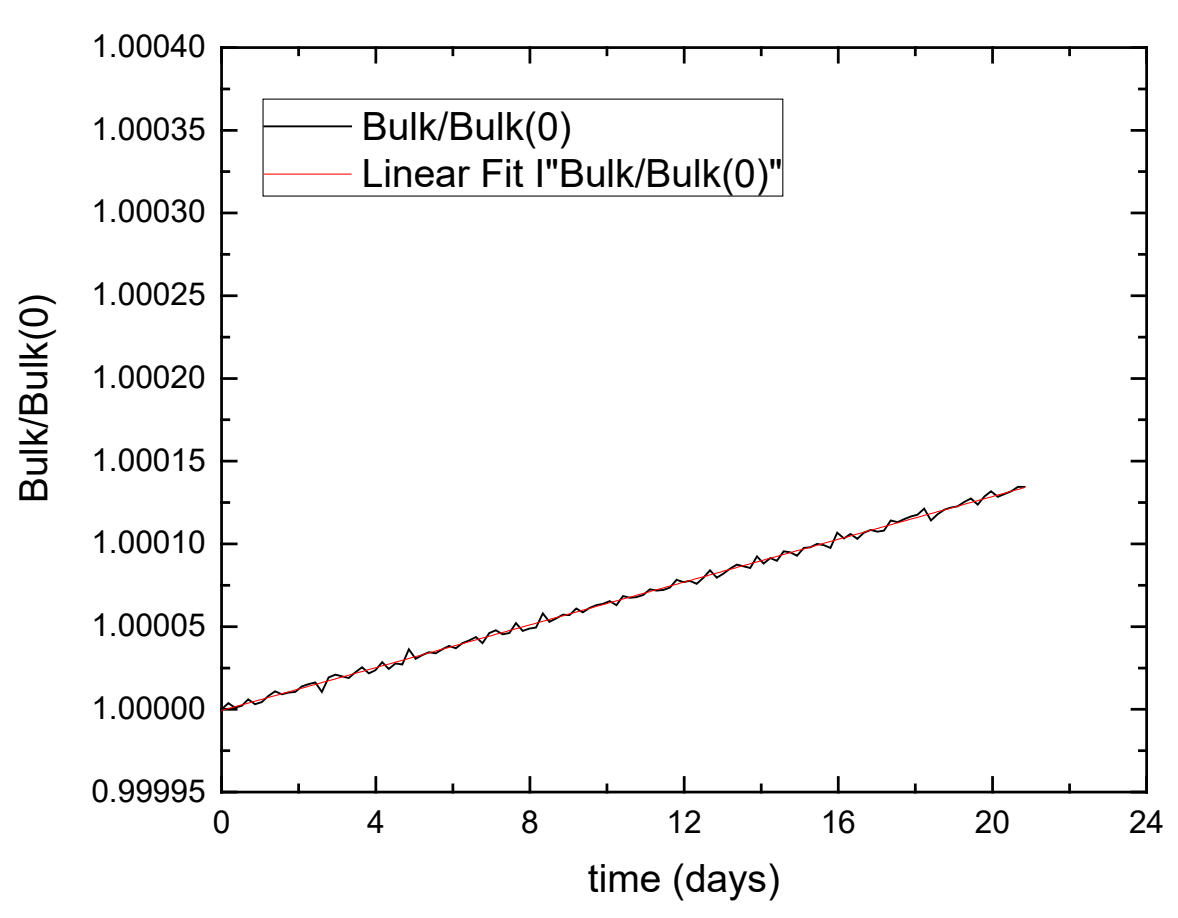

- This yields a density increase of about 600 ppm/yr opposite current thinking. 
We still do not know the number of defects per decay produced by the $U$ recoil flash heating:

- It is tempting to use the activation energy of the RUS slope and assign it to the defect energy but too many things are going on at once:

- Ga diffusion.

- Thermal production of defects.

- Self-annealing.

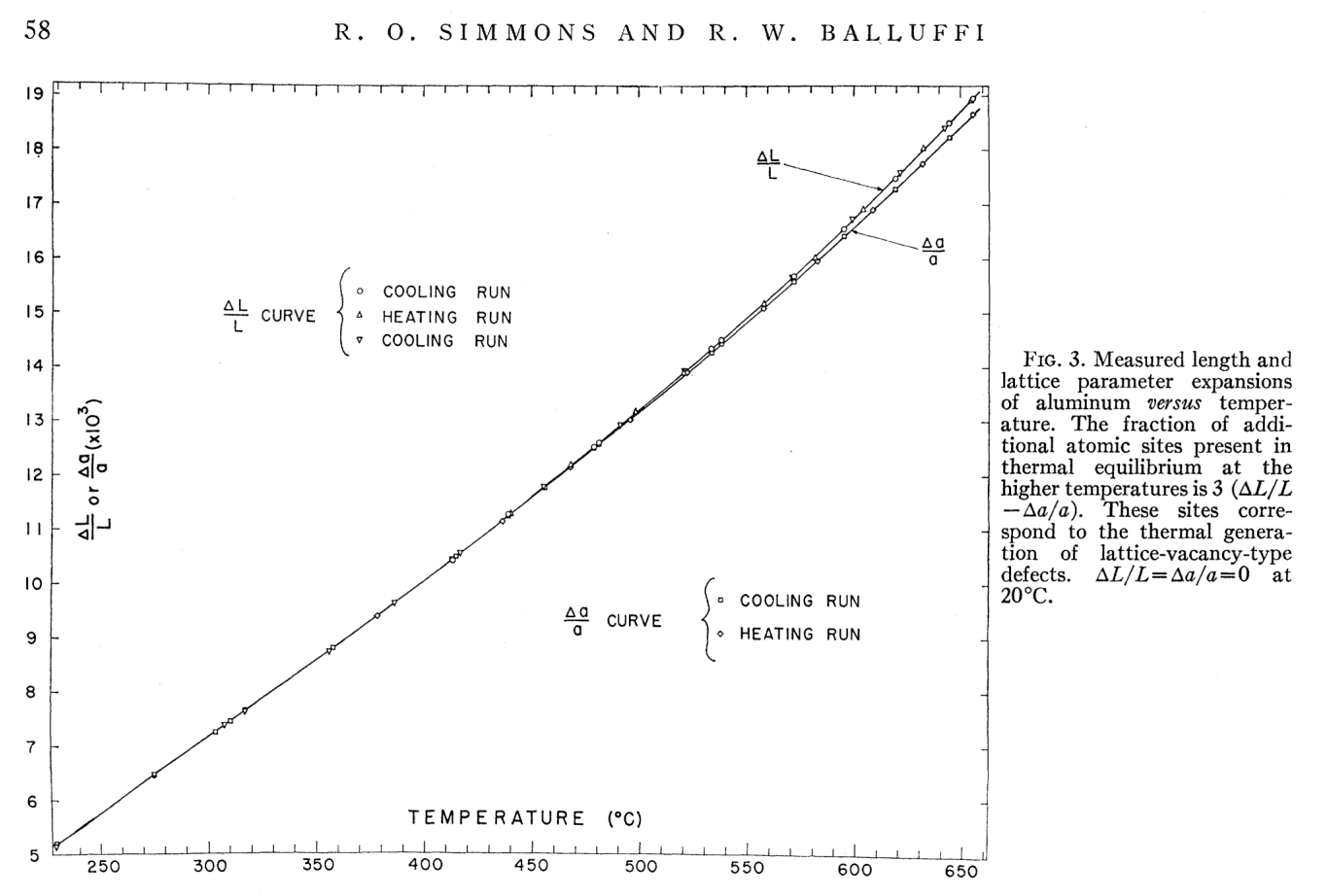




\section{Effects of radiation damage in $\delta$ - $\mathrm{Pu}$ (5)}

How can we determine the number of defects per decay or equivalently, the energy per defect, that produce the DSC results?

- It is tempting to use DFT supercell calculations but the unit cell volume for those calculations is fixed so calculations of the (testable) change in bulk modulus cannot be obtained.

- But we do know the total energy stored from DSC. So:

- If we can see the DSC time scale in RUS measurements of freshly-prepared material, because we know how many defects produce a given change in bulk modulus, and the total energy, we get the number.

- If we can see the DSC time scale in dilatometer measurements of freshlyprepared material, because we know how many defects produce a given change in density, and the total energy, we get the number.

- Can we use the diffuse background in diffraction, pdf, or EXAFS to see the DSC time scale and get the number that way? Hard to do.

- Can we correct historical data for thermal buoyancy? 
Implications:

- If we confirm our predictions then we know the size we compute for defects is correct.

- We know that the thermally-recoverable defects saturate in a few months.

- The $30 \mathrm{ppm} / \mathrm{yr}$ of $U$ production will after many months, produce about $2000 \mathrm{ppm} / \mathrm{yr}$ of defect clusters and so they should start to overlap at about 500 years.

- But Americium etc. tend to make short bonds less stable so this will start to reduce the stiffening and densification effects after a few decades.

- Although the Pu is stiffening, it is also densifying, so there is pre-compression of plutonium as it ages, 
We have not succeeded in answering all of our questions. Indeed, we sometimes feel that we have not completely answered any of them. The answers we have found only served to raise a whole new set of questions. In some ways we feel that we are as confused as ever, but we think we are now confused on a higher level, and about more important things.

-Author unknown 